\title{
TITLE:
}

\section{Wave emission on interacting heterogeneities in cardiac tissue}

$\operatorname{AUTHOR}(\mathrm{S})$ :

Hörning, Marcel; Takagi, Seiji; Yoshikawa, Kenichi

\section{CITATION:}

Hörning, Marcel ... [et al]. Wave emission on interacting heterogeneities in cardiac tissue. Physical Review E 2010, 82(2): 021926.

\author{
ISSUE DATE: \\ 2010-08 \\ URL: \\ http://hdl.handle.net/2433/130688 \\ RIGHT: \\ (C) 2010 The American Physical Society
}


PHYSICAL REVIEW E 82, 021926 (2010)

\title{
Wave emission on interacting heterogeneities in cardiac tissue
}

\author{
Marcel Hörning, ${ }^{1, *}$ Seiji Takagi, ${ }^{3}$ and Kenichi Yoshikawa ${ }^{1,2}$ \\ ${ }^{1}$ Department of Physics, Graduate School of Science, Kyoto University, Kyoto, Japan \\ ${ }^{2}$ Spatio-Temporal Order Project, ICORP JST, Kyoto 606-8502, Japan \\ ${ }^{3}$ Research Institute for Electronic Science, Hokkaido University, Sapporo 060-0812, Japan \\ (Received 17 June 2010; revised manuscript received 27 July 2010; published 31 August 2010)
}

\begin{abstract}
Cardiac arrhythmias, a precursor of fibrillationlike states in the beating heart, are associated with spiral waves, which are likely to become pinned to heterogeneities. Far-field pacing (FFP) is a promising method for terminating such waves by using heterogeneities in the tissue as internal pacing sites. In this study we investigated the role of multiple obstacles and their interaction during FFP. We show that a secondary nearby obstacle can significantly modulate the minimum electrical field in FFP. Further, we show that essentially the same effect can be observed in cardiac tissue culture, which is a powerful experimental model to simulate heart activity. Here, an isotropic cell distribution leads to domain formation of locally distributed depolarization sites. Both secondary obstacles and domain formation of local depolarization sites can modulate energy requirements to originate wave propagation on obstacles. Our theoretical result was confirmed by experiments with cardiomyocyte monolayers. This result may be useful for the future application of FFP to a real beating heart.
\end{abstract}

DOI: 10.1103/PhysRevE.82.021926

PACS number(s): 87.18.Hf, 05.45.-a, 87.19.Hh

\section{INTRODUCTION}

Excitable media can exhibit spatiotemporal patterns such as single spiral waves and multispiral dynamics (spatiotemporal chaos). In cardiac tissue, these are called ventricular tachycardia (VT) and ventricular fibrillation (VF) leading often to fatal conditions and even death. Considerable research has been conducted to elucidate the origin of the formation of VT and VF $[1,2]$. However, much less attention has been devoted to understanding how to terminate spatiotemporal patterns, which can easily occur under certain circumstances [3]. Recent articles have proposed various mechanisms for terminating free and heterogeneity-bounded spiral waves in experimental [4-10] and theoretical frameworks [6-8,11-14]. High- frequency stimulation, also known as anti-tachycardia pacing (ATP), or even a single stimulus can be sufficient to control patterns such as VT. However, to recover VF in a beating heart, these approaches are insufficient or frequently fail due to the complex structure of the heart. A procedure that has been proposed to control VT is so-called Far-Field pacing (FFP). FFP is the application of a stimulus directly to the heart. This has the advantage that the applied stimulus can be up to two orders of magnitude lower than that with automated external defibrillators (AED), since the resistivity of the skin does not need to be considered [10].

A special case of FFP is the application of low current to induce wave emission on heterogeneities (WEH). This allows us to excite a region within the heart at local heterogeneities by applying a low electrical far-field. Since only low currents on the order of a few volts need to be applied [4], this is a promising method for terminating spatiotemporal chaos without damaging the heart, and it may be possible to

\footnotetext{
*Corresponding author; FAX: +81-75-753-3779; marcel@chem.scphys.kyoto-u.ac.jp
}

regulate the number and position of WEH wave-emitting sites by an electric field [8]. Until now, the termination of pinned spiral waves rotating on an obstacle has been demonstrated for single and independent distributed heterogeneities within numerical frameworks $[12,15,16]$ and in in vitro experiments of cardiac tissue culture [4]. However, little is still known regarding the application of FFP to VF in a real beating heart. Recently, it has been shown by Fenton et al. (2009) that fibrillated tissue can be successfully recovered by FFP with the use of field strengths that were only $13 \%$ of the energy required to cardiovert with a single shock. Importantly, cardioversion of fibrillation-like states in heart with the use of lower energies could reduce the pain and tissue damage associated with a large single shock and prolong the battery life of implantable devices [10].

In this study, we examined the effects of wave emission on obstacles under the application of an electrical far-field. We focus on the mutual influence of closely located obstacles and domains formed by induced membrane potential change in aligned and isotropically distributed cell tissue, respectively. Our findings suggest the possibility of the modulation of energy requirements to induce wave propagation on obstacles. Furthermore, we confirmed the numerical results obtained for the case of isotropic cell distribution by experiments of cardiac tissue culture [4-6].

\section{MATERIALS AND METHODS}

Numerical simulations of the realistic Beeler-Reuter (BR) model [17] were adapted by using aligned and isotropic cell distributions. Experiments on cardiac tissue culture were performed for comparison with the obtained numerical data.

\section{A. Numerical methods}

The ionic currents in the model were described by the BR model, and were integrated with the Crank-Nicolson method, 
as explained by Keener and Bogar (1998) [18]. The bidomain model equations are written in terms of intracellular and extracellular membrane potential, $\Phi_{i}$ and $\Phi_{e}$, respectively,

$$
\begin{gathered}
\nabla \cdot \sigma_{i} \nabla \Phi_{i}=\beta\left[C_{m} \frac{\partial}{\partial t}\left(\Phi_{i}-\Phi_{e}\right)+I_{i o n}\right]-I_{i}, \\
\nabla \cdot \sigma_{e} \nabla \Phi_{e}=-\beta\left[C_{m} \frac{\partial}{\partial t}\left(\Phi_{i}-\Phi_{e}\right)+I_{i o n}\right]-I_{e}
\end{gathered}
$$

where $\sigma_{i}$ and $\sigma_{e}$ are the conductivity tensors, and $I_{i}$ and $I_{e}$ are the externally injected currents, in intracellular and extracellular spaces, respectively. $C_{m}$ is the membrane capacitance per unit area of the cell membrane and $\beta$ is the ratio of membrane surface area to tissue volume. The ionic current is defined as $I_{i o n}=G_{m} \Phi$, where $G_{m}=0.165 \mathrm{mS} / \mathrm{cm}^{2}$ and the membrane potential $\Phi=\Phi_{i}-\Phi_{e}$. The BR model considers four currents: the time-independent and time-activated slow outward currents of potassium, the fast inward current of sodium and the slow inward current of calcium. These currents are controlled by 6 gating variables, $\Phi$, and the calcium concentration. All six gating variables $\left(x_{1}, m, h, j, d, f\right)$ follow the same type of ordinary differential equations of the form,

$$
\frac{\mathrm{d} m}{\mathrm{~d} t}=\frac{\bar{m}(\Phi)-m}{\tau_{m}(\Phi)},
$$

where the fast gating variable $m$ is treated adiabatically at each times step as $\bar{m}(\Phi)=m(t)$ to optimize the integration protocol as described by Efimov et al. (1995) [19] and Takagi et al. (2004) [15].

Sodium and calcium conductance were assumed to be $g_{N a}=2.4 \mathrm{mS} \mathrm{cm}^{-1}$ and $g_{C a}=0.045 \mathrm{mS} \mathrm{cm}^{-1}$, respectively $[11,15]$. The external conductivity is isotropic $\sigma_{e x}=\sigma_{e y}$ $=4 \mathrm{mS} \mathrm{cm}{ }^{-1}$, and the internal conductivity for the alignedcell distribution is $\sigma_{i x}^{a}=4 \sigma_{i y}^{a}=4 \mathrm{mS} \mathrm{cm}{ }^{-1}$, with an anisotropy ratio of 4 . Simulations were performed with a spatiotemporal step of $0.15 \mathrm{~mm}$ and $0.05 \mathrm{~ms}$ with a grid size of 200 $\times 200$ units.

Isotropically distributed cell tissue was simulated by defining the alignment angle $\theta_{x, y}$ randomly for each cell. The intracellular conductivity can be written as

$$
\begin{gathered}
\sigma_{i x}^{i}=\sigma_{i x}^{a} \cos \theta_{x, y}-\sigma_{i y}^{a} \sin \theta_{x, y}, \\
\sigma_{i y}^{i}=\sigma_{i x}^{a} \sin \theta_{x, y}+\sigma_{i y}^{a} \cos \theta_{x, y},
\end{gathered}
$$

where $\sigma_{i x}^{i}$ and $\sigma_{i y}^{i}$ are the projected conductivities induced parallel and perpendicular to the electrical field, respectively.

\section{B. Experimental methods}

Cell culture. Primary cell cultures of neonatal rat ventricular myocytes were prepared as described elsewhere [20,21]. Briefly, hearts were isolated from 2-day-old SPF Wistar rats, minced and treated with collagenase. After cells were collected by centrifugation, they where preplated for 1 h. Cells were collected and plated on 22-mm-diameter glass coverslips coated with fibronectin $(12 \mu \mathrm{g} / \mathrm{ml})$ at a cell density of $2.6 \times 10^{3}$ cells $/ \mathrm{mm}^{2}$ with plating medium (Dulbeccomodified Eagle Medium with $10 \%$ fetal bovine serum, $1 \%$ penicillin streptomycin). Thereafter, cells were incubated for $24 \mathrm{~h}$ in a humidified atmosphere at $37{ }^{\circ} \mathrm{C}$ and $5 \% \mathrm{CO}_{2}$. The medium was replaced by a contraction medium (minimum essential medium with $10 \%$ calf serum, $1 \%$ penicillin streptomycin) and cells were incubated under the same conditions. Obstacles were made of polydimethylsiloxane (PDMS) with a radius of between 0.5 and $2.3 \mathrm{~mm}$, and placed on a glass coverslip before coating of with fibronectin [13].

Observation. Experiments were performed 4 days after plating. Before each observation, cells were labeled with the $\mathrm{Ca}^{2+}$-sensitive fluorescent dye Fluo-8, and the medium was replaced with Tyrode solution at room temperature. Fluorescence was observed with an inverted microscope (IX-70; Olympus, Tokyo Japan) by the use of a $\times 2.0$ magnification objective lens (PLAPON, N.A. $=0.08$, Olympus) in combination with a $\times 0.35$ intermediate lens [22]. Raw images were obtained with an electron- multiplying CCD (iXon DV887ECS-UVB; Andor) with $64 \times 64$ pixels at a 14-bit resolution with 200 frames/s after $8 \times 8$ binning.

Electrical-field stimulation of tissue was applied using a parallel set of platinum wire electrodes with an interelectrode distance of $4 \mathrm{~cm}$. Bipolar rectangular pulses were delivered between 2.8 and $9.2 \mathrm{~V}$ with a pulse length of $10 \mathrm{~ms} / 10 \mathrm{~ms}\left(S^{10}\right)$ and $100 \mathrm{~ms} / 10 \mathrm{~ms}\left(S^{100}\right)$.

Data analysis. The details of the data analysis have been reported previously [5,23]. Data were processed by ImageJ [24] analysis software with custom plug-ins. The time series of each pixel were filtered using a Savitzky-Golay filter for noise reduction.

\section{RESULTS}

It has been shown in numerical and experimental models of cardiac tissue that a change in the cell-membrane potential at an obstacle (cellular cleft) can be induced by applying an electrical far-field [25]. This effect has been referred to as wave emission on heterogeneities (WEH), secondary sources, and virtual electrodes $[8,25,26]$. WEH can be briefly described as follows by considering cardiac tissue as a two phase, i.e., intracellular and extracellular, medium [18]. The electron flux inside the intracellular space that originates from the anode prevents flow on the boundary of an intracellular cleft (obstacle). That leads to a local decrease in the membrane potential, since current flows outward from the intracellular space into the extracellular space, and is measured as a positive transmembrane current. The outward flux is indicated illustratively as diagonal upward arrows in Fig. 1(a). This means that the membrane potential is decreasing (Ohm's law). Since the total current of both spaces is conserved, on the opposite side of the intracellular cleft an inward flux of current can be observed, indicated as diagonal downward arrows in Fig. 1(a), and this leads to a local increase in the membrane potential. This eventually leads to the origination of wave propagation, when a minimum electrical field, $E_{\text {min }}$, is applied. Generally, $E_{\text {min }}$ decreases with an increase in the length of the intracellular cleft, which is also 
(a)

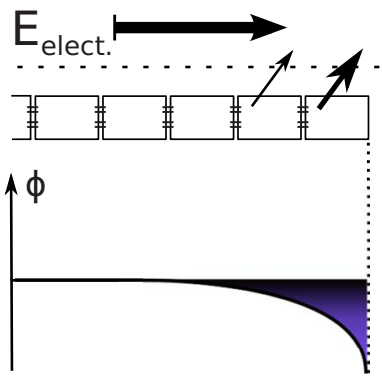

(b)

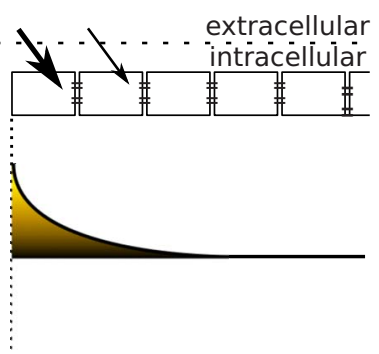

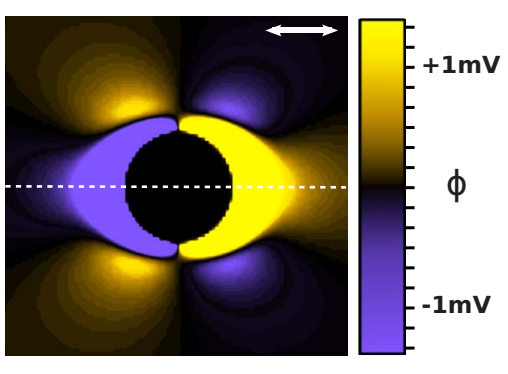

FIG. 1. (Color online) Schematic representation of wave emission on heterogeneities. The electrical far-field, $E_{\text {elec }}$, is applied as indicated by the upper arrow. $\Phi$ is the membrane potential, and depolarized and hyperpolarized regions are marked as yellow (white) and blue (gray), respectively. (a) The change in the membrane potential for cells nearby an intracellular cleft is illustrated. Diagonal arrows illustrate outward and inward transmembrane current, respectively. (b) shows induced patterns of excitation on a round obstacle (cellular cleft) by an electrical field in a bidomain model. The direction of cell alignment is indicated by the white double-headed arrow. Dipole and hexapole components are shown on and near the obstacle located at the center of the figure, respectively. The obstacle radius is $2.7 \mathrm{~mm}$. The profile of the white horizontal dashed line corresponds qualitatively to the membrane potential profile in (a).

valid for increasing radii of round obstacles in twodimensional tissue culture [8,25]. The applied electrical farfield induces dipole and hexapole patterns of excitation on the obstacle $[15,16]$. Depending on the strength of the stimulus, only the dipole components lead to the origination of waves in cardiac tissue [25]. Figure 1(b) shows the patterns induced by an electrical field in cardiac tissue on a round obstacle. The depolarized dipole component on the obstacle eventually leads to wave propagation when the excitation threshold and minimum area needed to originate waves are reached [27].

\section{A. Influence of secondary obstacles}

A secondary obstacle located close to another obstacle can lead to a change in $E_{\text {min }}$. Figure 2(a) shows an example in which two obstacles of the same size $\left(R_{1}=R_{2}=1.5 \mathrm{~mm}\right)$ are situated relative to each other so that the lower obstacle takes advantage of the upper obstacle. The lower obstacle shows wave propagation while the upper one does not. The applied electrical field is $E=430 \mathrm{mV} / \mathrm{cm}$, which is $15 \mathrm{mV} / \mathrm{cm}$ less than $E_{\text {min }}(R=1.5 \mathrm{~mm})$. For comparison, Fig. 2(b) shows two obstacles situated far from each other with an applied electrical field of $E=450 \mathrm{mV} / \mathrm{cm}$, which is slightly higher than $E_{\text {min }}(R=1.5 \mathrm{~mm})$.

The effect shown in Fig. 2(a) is due to the hexapole component of the upper obstacle, which overlaps the dipole component of the lower obstacle. Since the components overlap, $E_{\text {min }}$ for the lower obstacle is reduced depending on the strength of the hexapole component of the upper obstacle. In contrast, the upper obstacle remains uninfluenced or is eventually influenced by the hyperpolarized hexapole component of the lower obstacle. Figure 3(a) shows an illustration of two obstacles situated diagonally to each other in the same manner as in Fig. 2(a). The depolarized dipole of obstacle 1 and the depolarized hexapole of obstacle 2 overlap, which leads to a decrease in $E_{\text {min }}$ for obstacle 1. On the other hand, in Fig. 3(b), the depolarized dipole of obstacle 1 and the hyperpolarized dipole of obstacle 2 overlap and lead to an increase in $E_{\min }$ for obstacle 1. To analyze the effective (a)
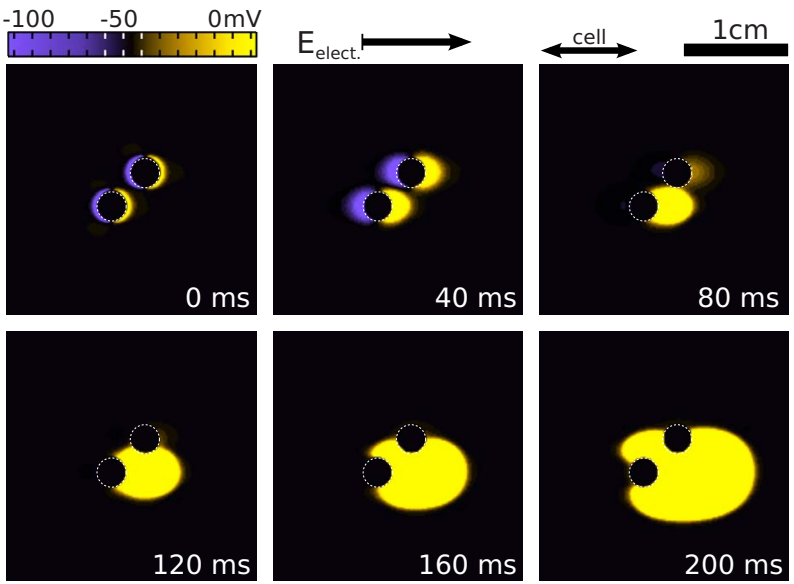

(b)
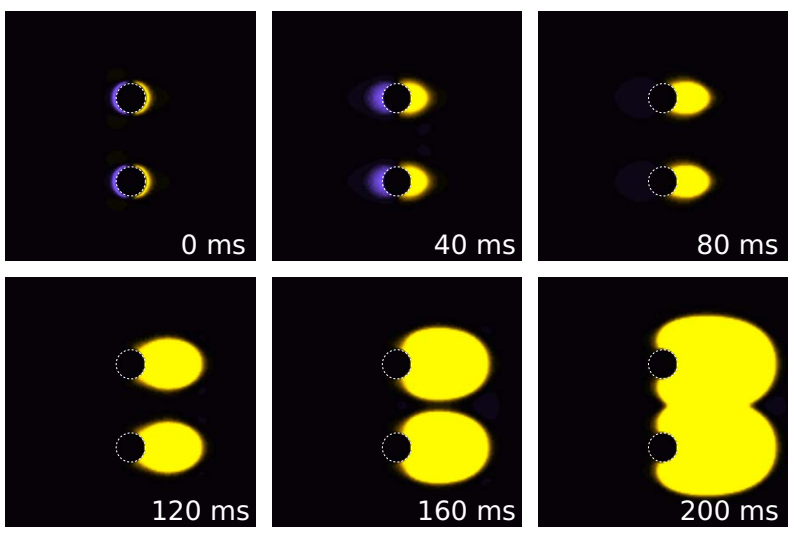

FIG. 2. (Color online) Neighboring obstacles under an applied electrical field. The radius of both obstacles is $1.5 \mathrm{~mm}$, and the respective critical electrical field is $E_{\text {min }}(R=1.5 \mathrm{~mm})$ $=445 \mathrm{mV} / \mathrm{cm}$. The direction of the applied electrical field is indicated by the arrow, and the direction of cell alignment is indicated by the double-headed arrow. (a) shows influenced obstacles. The applied electrical field is $E=430 \mathrm{mV} / \mathrm{cm}$. Only the lower obstacle shows wave propagation. (b) shows obstacles that are not influenced. The applied electrical field is $E=450 \mathrm{mV} / \mathrm{cm}$. 
(a)

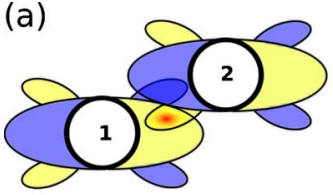

(b)

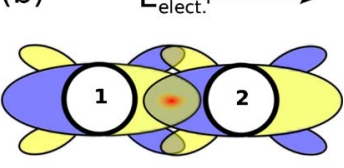

(c)

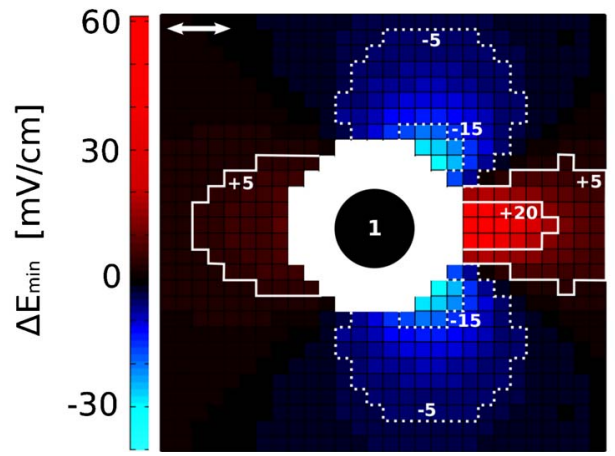

FIG. 3. (Color online) Effective electrical field of neighboring obstacles. (a) and (b) show illustrations of obstacle situations, with decreases and increases in $E_{\text {min }}$, respectively. Yellow (white) and blue (gray) indicate depolarized and hyperpolarized regions around the obstacles, respectively. (c) shows the change in the electrical field $\Delta E_{\min }$ for a $2.25 \mathrm{~mm}$-sized obstacle influenced by a $1.5 \mathrm{~mm}$ sized obstacle depending on their relative positions. The color indicates the change in $E_{\min }^{1}$ induced by the secondary obstacle: red shows an increase and blue shows a decrease in $E_{\min }^{1}(R$ $=2.25 \mathrm{~mm})=421 \mathrm{mV} / \mathrm{cm}$. Contours for decrease and increase in $E_{\text {min }}$ are drawn for -5 and $-15 \mathrm{mV} / \mathrm{cm}$, and for +7 and $+20 \mathrm{mV} / \mathrm{cm}$ by dotted and solid white lines, respectively. The white double-headed arrow indicates the direction of cell alignment.

change in $E_{\min }$ depending on the arrangement of the obstacles relative to each other, we determined $E_{\min }$ for an obstacle with radius $R_{1}=2.25 \mathrm{~mm}$ influenced by a smaller $R_{2}$ $=1.5 \mathrm{~mm}$-sized obstacle. The change of the minimum electrical field $\Delta E_{\min }=E_{\min }^{1}-E_{\min }^{1,2}$ was mapped as is shown in Fig. 3(c). The position of the larger obstacle $R_{1}$ was fixed at the center, and the smaller obstacle was positioned around the other by determining $\Delta E_{\min }$. The black color indicates that $\Delta E_{\min }=0 \mathrm{mV} / \mathrm{cm}$, and red and blue indicate an increase and decrease in $E_{\min }^{1,2}$, respectively. $E_{\min }$ increases when the obstacles are aligned, as illustrated in Fig. 3(b), and $E_{\min }$ decreases when the obstacles are diagonal to each other, as illustrated in Fig. 3(a). In contrast, when the obstacles are positioned parallel to each other or are far from each other, no significant effect is observed.

\section{B. Effects of cell orientation}

The results presented in the previous section are strongly related to the effect of cell orientation. This is due to the fact that no hexapole component can be induced when the cells are not parallel. However, nonaligned cells, where the cells have a random orientation, as in the experimental model system of cardiac tissue culture, show the effect of domain building throughout the tissue due to discontinuities of conductivity in the tissue [10]. Figure 4 shows the effect of an applied electrical far field on randomly distributed celloriented tissue with an obstacle in the center of the tissue.

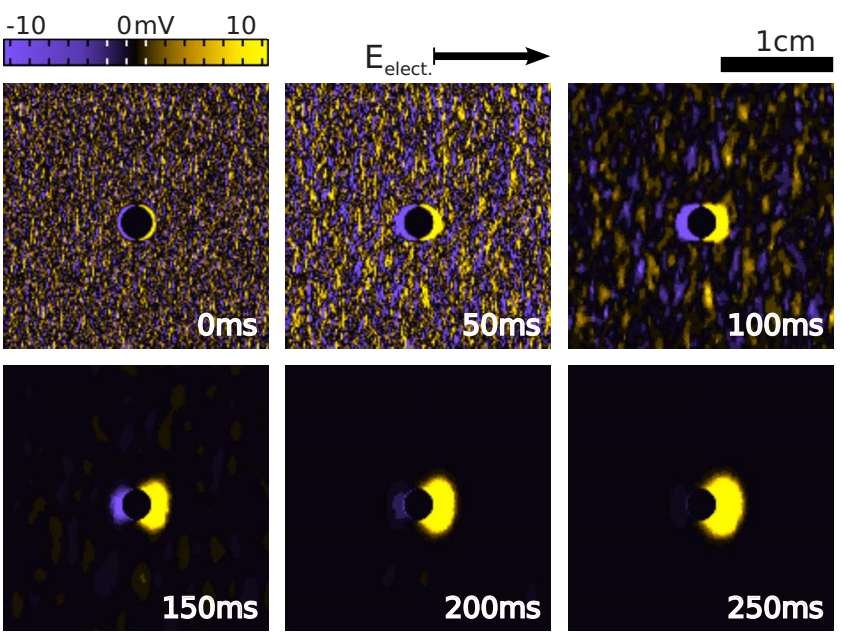

FIG. 4. (Color online) Effect of WEH in isotropically distributed cell tissue. Cells are randomly orientated [see Eq. (5)]. The obstacle radius is $1.5 \mathrm{~mm}$, and $E_{\min }$ is $550 \mathrm{mV} / \mathrm{cm}$

The electrical shock induces small, locally distributed dipoles at the time of field application, which lead to small domains after $50 \mathrm{~ms}$, and subsequently to bigger domains with vanishing micro domains at $100 \mathrm{~ms}$. After $150 \mathrm{~ms}$, the applied electrical shock-induced domains vanish almost completely, and only the depolarization induced on the obstacle leads to wave propagation.

Not every applied stimulus leads to wave propagation. Whether or not excitation at the obstacle leads to a propagating wave depends on both the constellation of cells and domain creation in the proximity of the obstacle. When a hyperpolarized region is in front of the obstacle, it might hinder wave propagation. The only way to overcome this barrier is to apply a stronger electrical field. On the other hand, when a depolarized domain exists in front of the obstacle, it eventually supports wave propagation, so that even a weaker electrical field would be sufficient. The influence of the domains is comparable to the effect of a secondary obstacle, as shown in the previous section.

The dependency of $E_{\min }$ for isotropically distributed cell tissue is shown in Fig. 5. Figure 5(a) shows the probability of wave propagation depending on the obstacle size. As the obstacle becomes smaller, $E_{\min }$ increases and the sigmoidal distribution becomes broader. The figure shows obstacles with radii of from $8 \mathrm{~mm}$ to $30 \mathrm{~mm}$. In Fig. 5(b), the 50\% probability of wave propagation is shown with error bars and is approximated with a solid line. The error range indicates a $10 \%$ probability of wave propagation. For comparison, $E_{\min }$ for an aligned-cell distribution is also shown. When the electrical field is applied parallel to the cell alignment, $E_{\min }^{\|} \operatorname{cor}$ responds to the lower dashed line, and when it is applied perpendicular, $E_{\min }^{\perp}$ corresponds to the upper dashed line. $E_{\min }$ for larger obstacles is comparable to $E_{\text {min }}^{\|}$. In contrast, smaller obstacles tend to give much stronger $E_{\min }$, but this is still much weaker than $E_{\text {min }}^{\perp}$.

\section{EXPERIMENTAL RESULTS}

Cardiomyocyte monolayers were used to verify the obtained numerical data. We particularly focused on the rela- 
(a)

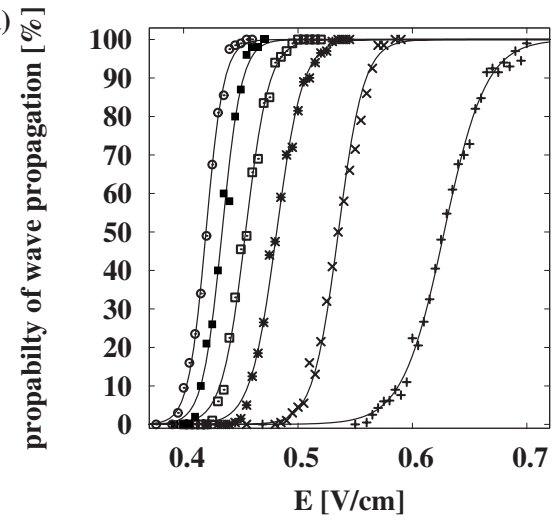

(b)

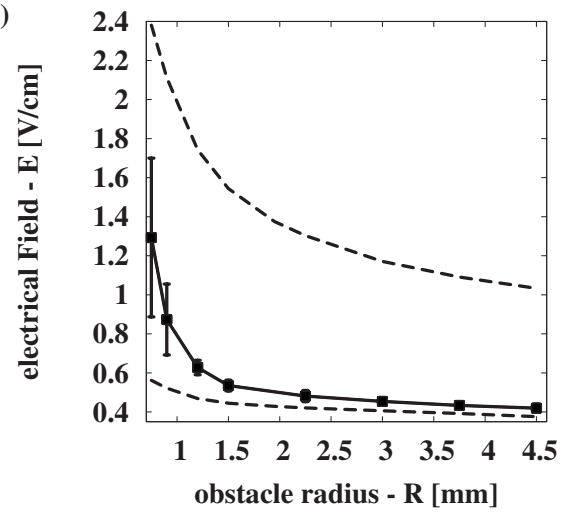

FIG. 5. Comparison of minimum electrical fields. (a) Probability of wave origination depending on obstacle size and electrical field. The distribution is plotted for obstacles of size 4.0, 3.75, 3.0, 2.25, 1.5 , and $1.2 \mathrm{~mm}$, corresponding to the distribution plotted from left to right, respectively. Solid lines are sigmoidal functions fitted to the respective obstacle size. Each data point represents at least 200 simulations. The upper and lower dashed lines in (b) show $E_{\min }^{\perp}$ and $E_{\min }^{\|}$for aligned-cell tissue, respectively. For isotropic distributed cell tissue, 50\%-wave probabilities are plotted as data points and the range of $10 \%$-wave probability is plotted as error bars.

tionship between the electrical field and obstacle radius [see Fig. 5(b)] for the estimation of $E_{\min }^{\perp}$ by observing $E_{\text {min }}$ for isotropic cell distributions in two-dimensional cardiac tissue. We also examined the relationship between the stimulus duration and the minimum electrical field to obtain $\mathrm{WEH}$.

To determine the obstacle size and $E_{\min }$ in cardiac tissue, 64 monolayer preparations containing a single obstacle 1.0 to $4.6 \mathrm{~mm}$ in diameter were observed. $E_{\min }$ was obtained by applying electrical fields from 0.7 to $2.5 \mathrm{~V} / \mathrm{cm}$ in steps of 25 $\mathrm{mV} / \mathrm{cm}$, which corresponds to an applied voltage of $0.1 \mathrm{~V}$ at electrodes $4 \mathrm{~cm}$ apart. Electrical stimuli were applied with a period of $2 \mathrm{~s}$. The effect of WEH was confirmed when 3 subsequent stimuli originated from the obstacle to ensure the reliability of the observed WEH effect, since spontaneous wave emission that is triggered by low-field stimuli can be observed. The procedure was repeated on each monolayer for bipolar rectangular stimuli of $+100 \mathrm{~ms} /-10 \mathrm{~ms}\left(S^{100}\right)$ and $+10 \mathrm{~ms} /-10 \mathrm{~ms}\left(S^{10}\right)$.

Figure 6(a) shows an example of WEH observed in experiments on cardiac tissue culture with electric field stimulation at $1.2 \mathrm{~V} / \mathrm{cm}$ with a rectangular bipolar stimulus of $S^{100}(100 \mathrm{~ms})$ on a $1.85 \mathrm{~mm}$-diameter obstacle. The induced (a)

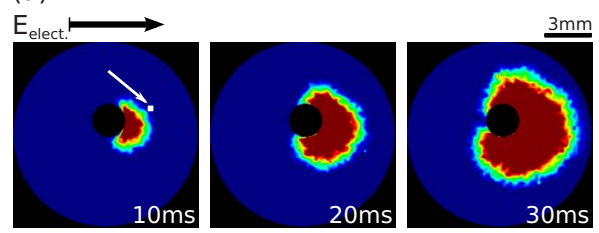

(b)

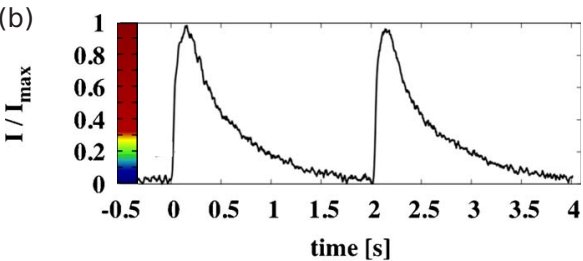

(c)

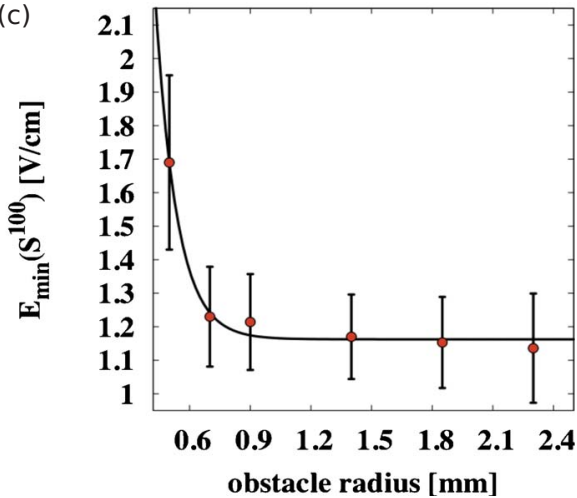

FIG. 6. (Color online) Experiments on the minimum electrical far-field of WEH in cardiac tissue culture. (a) Snapshots of wave origination on the right side of the obstacle. Electrical-field stimulation at $1.2 \mathrm{~V} / \mathrm{cm}$ was applied to a $1.85 \mathrm{~mm}$-diameter obstacle. The anode and cathode are placed $4 \mathrm{~cm}$ from each other on the left and right sides of the observation chamber, respectively. The field of view is $11.6 \mathrm{~mm}$. (b) The intensity profile recorded at the white mark emphasized by the white arrow in the first frame in (a). The intensity is scaled to the maximum intensity, $I_{\max }$. (c) $E_{\min }$ for a stimulation duration of $100 \mathrm{~ms}$ depending on the obstacle size. Equation (6) is plotted as a solid line.

wave propagation originates at the right side of the obstacle, which is located in the center of the tissue. Frames are shown at time steps of $10 \mathrm{~ms}$. Figure 6(b) shows the intensity profile recorded at the white mark indicated by the white arrow in the first frame of Fig. 6(a). Periodic field stimulation is applied at 0 and $2 \mathrm{~s}$. Black arrows indicate the time span and the color bar shows the respective intensity of excitation in the snapshots shown in Fig. 6(a). A decrease in the applied electrical far-field intensity eventually leads to a failure of wave propagation, where the minimum electrical field $E_{\text {min }}$ depends on the obstacle radius. Figure 6(c) shows the relationship between the obstacle radius $R$ and $E_{\min }$ for stimuli of $S^{100}$ under far-field stimulation. Data points show the average and standard deviation of $E_{\min }$ for each observed obstacle size. The dependency of $E_{\text {min }}(R)$ shows an exponential increase for a decrease in the obstacle radius, which can be approximated as

$$
E_{\text {min }}=E_{s} e^{-R / \kappa}+E_{0, \min },
$$

where $E_{s}=59.5 \mathrm{~V} / \mathrm{cm}$ and $\kappa=0.11 \mathrm{~mm}$ are constants of the system, $R$ is the radius of the obstacle, and $E_{0, \min }$ 

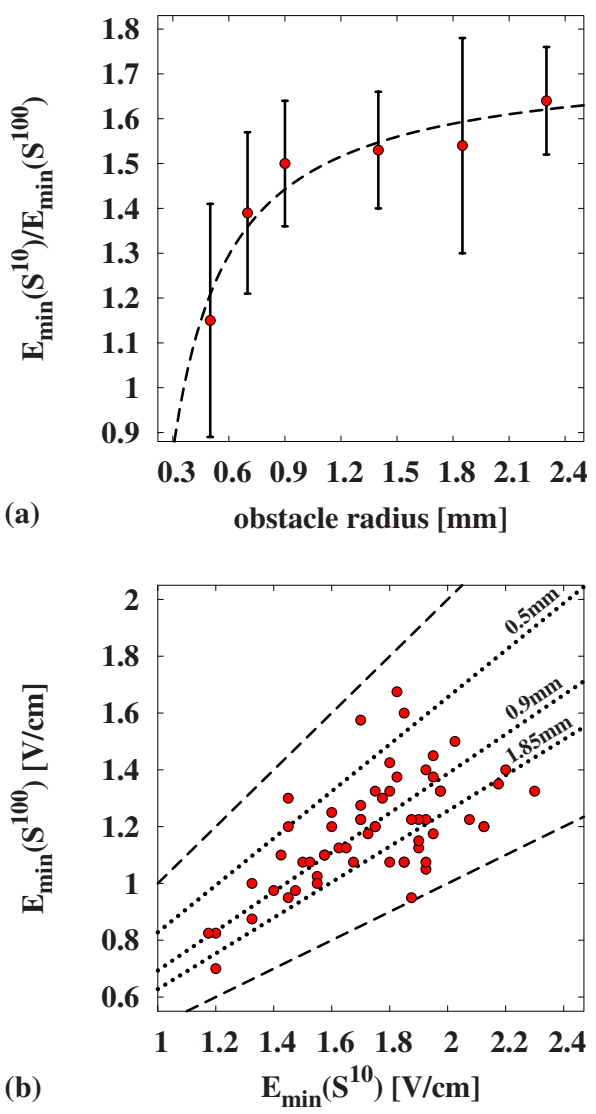

FIG. 7. (Color online) Comparison of the stimulation duration for the minimum electrical far-field in experiments with cardiac tissue. (a) Relationship between obstacle radius $R$ and the fraction of $E_{\text {min }}$ for stimulation durations of $10 \mathrm{~ms}$ and $100 \mathrm{~ms}$. (b) Relationship between $E_{\text {min }}\left(S^{10}\right)$ and $E_{\text {min }}\left(S^{100}\right)$ corresponding to stimulation durations of $10 \mathrm{~ms}$ and $100 \mathrm{~ms}$, respectively. The dotted lines correspond to the slopes of the obtained approximation in (a) for constant radii of $0.5,0.9$, and $1.85 \mathrm{~mm}$, respectively. The upper dashed line is the reference for $E_{\min }\left(S^{10}\right)=E_{\min }\left(S^{100}\right)$, and the lower dashed line corresponds to a slope of 2 .

$=1.16 \mathrm{~V} / \mathrm{cm}$ is the minimum electrical field for $R \rightarrow \infty$. Equation (6) is illustrated in Fig. 6(c) as a black solid line.

The correlation between applied stimulation pulses of $E_{\text {min }}\left(S^{10}\right)$ and $E_{\text {min }}\left(S^{100}\right)$ with respect to the obstacle radius is shown in Fig. 7(a). Although the error is rather large, a reciprocal decrease is observed for decreasing obstacle size. This means that, for smaller obstacle sizes, the difference between $E_{\text {min }}\left(S^{10}\right)$ and $E_{\text {min }}\left(S^{100}\right)$ decreases. The black dashed line is an approximation, which can be written as

$$
\frac{E_{\text {min }}\left(S^{10}\right)}{E_{\text {min }}\left(S^{100}\right)}=-d \cdot R^{-1}+C,
$$

where $R$ is the radius of the obstacle, $d=-0.26 \mathrm{~mm}$ is a scaling variable, which is approximately the size of a cardiac cell, and $C=1.74$ is a constant. Thus, for obstacles with $R$ $\rightarrow \infty$ (flat boundaries) the electrical far-field needed to originate wave propagation with a stimulation duration of $100 \mathrm{~ms}$ is approximately $75 \%$ lower than that with a stimulation duration of $10 \mathrm{~ms}$ in a two-dimensional monolayer of cardiac tissue culture. In addition, Fig. 7(b) shows the distribution of $E_{\text {min }}$ with respect to stimulation durations of $10 \mathrm{~ms}$ and 100 $\mathrm{ms}$ with round data points. The sloping dotted lines correspond to the approximations obtained for radii of $0.5,0.9$, and $1.85 \mathrm{~mm}$ in Fig. 7(a) (black dashed line). For reference, the figure shows $E_{\text {min }}\left(S^{10}\right)=E_{\text {min }}\left(S^{100}\right)$, which corresponds to a slope of 1, and a slope of 2 as the upper and lower dashed lines, respectively. The data points are widely distributed, since the natural variability of cardiomyocyte monolayers prohibits greater accuracy $[4,13,28]$. However, these findings are statistically comparable to the results of the numerical simulations.

\section{CONCLUSION}

We have demonstrated that, in both numerical simulations with the Beeler-Reuter model and experiments with cardiomyocyte monolayers, the minimum electrical field for observing WEH is strongly influenced by nearby obstacles in aligned- cell cardiac tissue, and depends on domain formation in isotropically distributed cell tissue culture.

In aligned-cell tissue, nearby obstacles can decrease or increase the minimum electrical field for originating wave propagation. When a secondary obstacle is located in front of the main obstacle, where the wave originates, $E_{\text {min }}$ of the main obstacle is increased. When the obstacle is placed diagonally in front of the main obstacle, $E_{\min }$ is decreased due to overlapping of the polarized dipole and hexapole of the main and secondary obstacles, respectively. In isotropically distributed cell tissue, no hexapole component is observed. However, domain formation throughout the tissue can lead to a change in $E_{\min }$ for the respective obstacle size, since domains located near the obstacle modulate $E_{\text {min }}$. Low depolarization and hyperpolarization domains are induced due to local discontinuities in conductivity. The probability of WEH follows a sigmoidal distribution for a constant obstacle size and increases with an increase in obstacle size. $E_{\text {min }}$ of isotropically distributed cell tissue is between $E_{\text {min }}^{\|}$and $E_{\text {min }}^{\perp}$.

For comparison with the results obtained numerically, experiments were performed with isotropically distributed cells in cardiac tissue culture. The results showed that $E_{\text {min }}$ increased monotonously with an increase in the obstacle radius according to Eq. (6). This result leads to the assumption that an increase in the obstacle size minimizes the influence of domain formation, since this was also observed in numerical simulations, so that $E_{0, \min } \rightarrow E_{\text {min }}^{\perp}$. On the other hand, with a decrease in obstacle size to single cardiac cells, $E_{\text {min }}$ approaches the same electrical field strength that is required to induce wave propagation through discontinuities in conductivity in cardiac tissue. If we consider the strong natural variability of cardiac tissue culture, we can estimate that $E_{0, \min }$ for a stimulation duration of $100 \mathrm{~ms}$ is approximately $1 \mathrm{~V} /$ $\mathrm{cm}$. Further, we have shown experimentally that, when the stimulation duration is decreased from $100 \mathrm{~ms}$ to $10 \mathrm{~ms}, E_{\text {min }}$ decreases with a decrease in the obstacle size according to Eq. (7). For large obstacles, $E_{\text {min }}\left(S^{100}\right)$ is roughly 2 times smaller than $E_{\text {min }}\left(S^{10}\right)$. However, for smaller obstacles close to the size of a cardiac cell, the difference in the stimulation strength decreases and becomes minor. While a pulse dura- 
tion of $1000 \mathrm{~ms}$ or longer leads to WEH, it also leads to a drastic increase in the number of pacing sites distributed throughout the tissue, which makes it difficult to distinguish between WEH on obstacles and WEH induced by discontinuities in conductivity in experiments with cardiac tissue culture. This suggests that an increase in the stimulation duration decreases the required stimulation strength and can thus decrease energy requirements of the implantable device and the pain of the patient.

Although the results in Fig. 3 suggests that a modulation of the orientation of anode and cathode of the implantable device could decrease the energy requirements to generate wave propagation, the effect of cell orientation plays a dominant role in the determination of the required electrical field strength (see Fig. 5). The effect of cell orientation can affect $E_{\text {min }}$ up to $2000 \mathrm{mV} / \mathrm{cm}$ depending upon the size of the obstacle. Thus, it is preferable to orient the anode and cathode in such a way that the applied electric field is parallel to the cell orientation, thereby minimizing both energy requirements and patient discomfort.

Unfortunately, optimizing the orientation of the electrode is not practically applicable, since the heart is a threedimensional object and the cell orientation is aligned at the wall of heart. This makes it impossible to have an optimized position of the electrode. To overcome this problem either one could try to use artificial obstacles of larger size, so that higher painful shocks are not required as Figs. 5 and 6(c) suggest, or alternatively a multielectrode device could be applied as it was already introduced by Fenton and co-workers. They have successfully shown that low- amplitude shocks delivered via two field electrodes were capable of terminating atrial fibrillation in isolated perfused canine atria [10].

Our results indicate that heterogeneities in cardiac tissue strongly influence each other under the application of low electrical far-fields (WEH). The size of the heterogeneity plays a major role not only in the minimum electrical field necessary to originate wave propagation, but also in the degree of influence of nearby heterogeneities or discontinuities in conductivity. This result may contribute to the improved application of WEH, especially in older patients with fibrosis and scarring, which are accompanied by a higher incidence of conductivity discontinuities [10,29].

\section{ACKNOWLEDGMENT}

This research was supported in part by JSPS (Grant No. 21-102).
[1] F. Fenton, E. Cherry, H. Hastings, and S. Evans, Chaos 12, 852 (2002).

[2] B. Echebarria and A. Karma, Phys. Rev. E 76, 051911 (2007).

[3] A. Pertsov, J. Davidenko, J. Salomonsz, W. Baxter, and J. Jalife, Circ. Res. 72, 631 (1993).

[4] J. Cysyk and L. Tung, Biophys. J. 94, 1533 (2008).

[5] A. Isomura, M. Hörning, K. Agladze, and K. Yoshikawa, Phys. Rev. E 78, 066216 (2008).

[6] M. Hörning, A. Isomura, K. Agladze, and K. Yoshikawa, Phys. Rev. E 79, 026218 (2009).

[7] M. Tanaka, A. Isomura, M. Hörning, H. Kitahata, K. Agladze, and K. Yoshikawa, Chaos 19, 043114 (2009).

[8] A. Pumir, V. Nikolski, M. Hörning, A. Isomura, K. Agladze, K. Yoshikawa, R. Gilmour, E. Bodenschatz, and V. Krinsky, Phys. Rev. Lett. 99, 208101 (2007).

[9] K. Agladze, M. Kay, V. Krinsky, and N. Sarvazyan, Am. J. Physiol. Heart Circ. Physiol. 293, H503 (2007).

[10] F. Fenton, S. Luther, E. Cherry, N. Otani, V. Krinsky, A. Pumir, E. Bodenschatz, and J. R. F. Gilmour, Circulation 120, 467 (2009).

[11] A. Pumir and V. Krinski, J. Theor. Biol. 199, 311 (1999).

[12] P. Bittihn, A. Squires, G. Luther, E. Bodenschatz, V. Krinsky, U. Parlitz, and S. Luther, Philos. Trans. R. Soc. London, Ser. A 368, 2221 (2010).

[13] M. Hörning, A. Isomura, Z. Jia, E. Entcheva, and K. Yoshikawa, Phys. Rev. E 81, 056202 (2010).
[14] V. Zykov, G. Bordyugovb, H. Lentz, and H. Engel, Physica D 239, 797 (2010).

[15] S. Takagi, A. Pumir, D. Pazo, I. Efimov, V. Nikolski, and V. Krinski, J. Theor. Biol. 230, 489 (2004).

[16] S. Takagi, A. Pumir, D. Pazo, I. Efimov, V. Nikolski, and V. Krinsky, Phys. Rev. Lett. 93, 058101 (2004).

[17] G. Beeler and H. Reuter, J. Physiol. 268, 177 (1977).

[18] J. Keener and K. Bogar, Chaos 8, 175 (1998).

[19] I. Efimov, V. Krinsky, and J. Jalife, Chaos 5, 513 (1995).

[20] T. Harada and A. Isomura, Prog. Theor. Phys. 161, (Suppl.), 107 (2006).

[21] S. Matoba, T. Tetsuya, N. Keira, A. Kawahara, and K. Akashi, Circulation 99, 817 (1999).

[22] E. Entcheva and H. Bien, Prog. Biophys. Mol. Biol. 92, 232 (2005).

[23] S. M. Hwang, K. H. Yea, and K. J. Lee, Phys. Rev. Lett. 92, 198103 (2004).

[24] W. Rasband, http://rsb.info.nih.gov/ij/

[25] V. Fast, A. G. S. Rohr, and A. Kleber, Circ. Res. 82, 375 (1998).

[26] M. G. Fishler, J. Cardiovasc. Electrophysiol. 9, 384 (1998).

[27] A. Winfree, Physica D 12, 321 (1984).

[28] Z. Lim, B. Maskara, F. Aguel, R. Emokpae, and L. Tung, Circulation 114, 2113 (2006).

[29] M. Spach and P. Dolper, Circ. Res. 58, 356 (1986). 